\title{
Curvaton reheating in non-minimal derivative coupling to gravity: NO models
}

\author{
Ramón Herrera* and Joel Saavedrat \\ Instituto de Física, Pontificia Universidad Católica de Valparaíso, \\ Av. Brasil 2950, Valparaíso, Chile. \\ Cuauhtemoc Campuzandi \\ Departamento de Física, Facultad de Física e Inteligencia Artificial, \\ Universidad Veracruzana, 91000, Xalapa Veracruz, México
}

(Dated: November 14, 2018)

\begin{abstract}
The curvaton reheating mechanism in a non-minimal derivative coupling to gravity for any nonoscillating (NO) model is studied. In this framework, we analyze the energy density during the kinetic epoch and we find that this energy has a complicated dependencies of the scale factor. Considering this mechanism, we study the decay of the curvaton in two different scenarios and also we determine the reheating temperatures. As an example the NO model, we consider an exponential potential and we obtain the reheating temperature indirectly from the inflation through of the number of e-folds.

PACS numbers: $98.80 . \mathrm{Cq}$
\end{abstract}

\footnotetext{
*Electronic address: ramon.herrera@pucv.cl

${ }^{\dagger}$ Electronic address: joel.saavedra@pucv.cl

${ }^{\ddagger}$ Electronic address: ccvargas@uv.mx
} 


\section{INTRODUCTION}

Einstein's gravity has been considered for many years as the physics of the universe and the standard model of the modern cosmology is based on this theory. However, also it is well known if we are searching for one complete description of the universe we need to use complements, in particular we are using different kind of matter in order to do an approximate description of the actual universe. For example at the early universe we need to introduce the inflationary paradigm in order to solve some problem of the standard model, and it is introduced the inflaton field in order to have the adequate expansion, and perturbation as seed of the large structure of the universe [1, 2]. From the current observation we need to introduce the dark energy in order to do an approximation to the observational data and touch the property theoretical description of our currently observations. We can continue doing this addition of elements or complement to the standard cosmological model in order to solve problems in the prediction, in some cases, or to fit with the more and more precise astronomical data [3, 4]. Alternative, we can change the background of the theory and we could consider modification to the Einstein's theory of gravity, as was done in tensorscalar theories or Jordan-Brans-Dicke theory [5]. On the other hand, we can use actions from higher dimensional theories or string theory, as the effective theory in the low energy limit [6]. Also, we could do the modification of the theory or the action, in a straightforward way, using a variational principle more general, for example

$$
S=\int d^{4} x \sqrt{-g}\left\{F\left(R, R_{\mu \nu} R^{\mu \nu}, R_{\mu \nu \lambda \rho} R^{\mu \nu \lambda \rho}, . .\right)+K\left(\phi, \partial_{\mu} \phi \partial^{\mu} \phi, \square^{2} \phi, R^{\mu \nu} \partial_{\mu} \phi \partial_{\nu} \phi, . .\right)-V(\phi)\right\},
$$

where $F$ and $K$ are arbitrary functions of the corresponding variables. This action implies different consequences, and we need to have in mind the basic principles of physics and therefore gravity. The consequences can be see direct in the equation of motions, we must ask a priori (according to the basic principles), a covariant formulation of equation of motion, the dynamics it is driven by second order differential equation, and it must satisfy the correspondence principle. However, the non-linear function $F$ and $K$ provided the general invariant that can meet at least two of this requirement, unfortunately we must deal with higher

order differential equation as equation of motion. Of course here we have matter described by a scalar field, the way as this was introduced in Einstein's gravity through the minimal 
coupling, between geometry and matter. In the action (1), we are considering in $K$ function the more general no-minimal coupling between the scalar field and gravity. Of course this new coupling modify the usual Klein-Gordon equation, and therefore the field equation for the scalar field is not longer a second order differential equation in this general case, as an example of this higher dynamics see Refs. [7-9]. It is clear that the modification of gravity in this way can be done by modification of the geometry $F\left(R, R_{\mu \nu} R^{\mu \nu}, R_{\mu \nu \lambda \rho} R^{\mu \nu \lambda \rho}, \ldots\right)$ or modification of matter sector $K\left(\phi, \partial_{\mu} \phi \partial^{\mu} \phi, \square^{2} \phi, R^{\mu \nu} \partial_{\mu} \phi \partial_{\nu} \phi, \ldots\right)$, this last choice we would like to discuss in more details. Currently there are a growing interest in the called Horndeski Lagrangian [10] the more general scalar field Lagrangian with non-minimal couplings between the scalar field and the curvature, and at the same time producing second order motion equations. In Ref. [11], was found that the equation of motion for the scalar field can be reduced to second order differential equation, when it is kinetically coupled to the Einstein tensor, $G^{\mu \nu} \partial_{\mu} \phi \partial_{\nu} \phi$, and in Ref. [12] the author investigated the cosmological scenarios for this kind of coupling. In this case the action is described by[11]

$$
S=\int d^{4} x \sqrt{-g}\left(\frac{R}{16 \pi G}-\frac{1}{2}\left(g_{\mu \nu}-\frac{1}{M^{2}} G_{\mu \nu}\right) \partial^{\mu} \phi \partial^{\nu} \phi-V(\phi)\right)+S_{m a t t e r}
$$

where $g$ corresponds to the determinant of the space-time metric $g_{\mu \nu}, R$ is the Ricci scalar and $G^{\mu \nu}=R^{\mu \nu}-\frac{1}{2} R g^{\mu \nu}$ is the Einstein tensor. Here the parameter $M$ is a constant with dimension of mass, and $V(\phi)$ corresponds to the effective potential associated to the scalar field $\phi$. The parameter $M^{-2}$ and its sign plays a critical role in this type of theory. Recently in Ref.[13] was studied the screening Horndeski cosmologies in which the ghost-free cosmological solutions occur if the parameter $M^{-2}<0$. However, analyzing the dynamical stability in these solutions was found that they are stable in the future but unstable in the past (initial spacetime singularity). In Ref.[14] was suggested that the parameter $M^{-2}>$ 0 in order to evade the ghost presents in the model. Also in Ref.[15] was shown that independently of the value of $M^{-2}$ the model does not present instabilities. In this form, we mention that the sign of $M^{-2}$ positive or negative is still an open issue in the literature, in particular for the early universe. In the following we will consider the value $M^{-2}>0$ in order to study the early universe. However, we mention that for negative values of the parameter $M^{-2}$, we would have to add the condition $1 \gtrsim H^{2} / M^{2}$, in order to evade possible imaginary quantities in our model. 
The equation of motions for the geometry from the variation of the metric $\delta g_{\mu \nu}$

$$
G_{\mu \nu}=8 \pi G\left(T_{\mu \nu}^{\phi}+T_{\mu \nu}^{\text {matter }}+\frac{1}{M^{2}} T_{\mu \nu}^{\text {derivatives }}\right)
$$

where $T_{\mu \nu}^{\text {matter }}$ is the usual energy momentum tensor for the matter, $T_{\mu \nu}^{\phi}=\nabla_{\mu} \phi \nabla_{\nu} \phi-$ $\frac{1}{2} g_{\mu \nu}(\nabla \phi)^{2}$ and the new component is given by

$$
\begin{aligned}
T_{\mu \nu}^{\text {derivatives }}= & -\frac{1}{2} \nabla_{\mu} \phi \nabla_{\nu} R+2 \nabla_{\alpha} \phi \nabla_{(\mu} \phi R_{\nu)}^{\alpha}+\nabla^{\alpha} \phi \nabla^{\beta} \phi R_{\mu \alpha \nu \beta}+\nabla_{\mu} \nabla^{\alpha} \phi \nabla_{\nu} \nabla_{\alpha} \\
& -\nabla_{\mu} \nabla_{\nu} \phi \square \phi-\frac{1}{2}(\nabla \phi)^{2} G_{\mu \nu}+g_{\mu \nu}\left(-\frac{1}{2} \nabla^{\alpha} \nabla^{\beta} \nabla_{\alpha} \nabla_{\beta}+\frac{1}{2}(\square \phi)^{2}-\nabla_{\alpha} \phi \nabla_{\beta} \phi R^{\alpha \beta}\right) .
\end{aligned}
$$

The variation of the action respect to the scalar field $\delta \phi$ gives its equation of motion

$$
\left(g^{\mu \nu}+\frac{1}{M^{2}} G^{\mu \nu}\right) \nabla_{\mu} \nabla_{\nu} \phi=\frac{d V}{d \phi} .
$$

In relation to the cosmological consequences in non-minimally derivative coupling to gravity were studied in Refs.[16, 17]. Also, the theory of the density perturbation in the early universe with this non-minimally derivative coupling was analyzed in Ref.[18].

On the other hand, the reheating of the universe is a procedure in which the scalar field or inflaton field is converted into the standard model particles[19]. During reheating of the universe, the best part of the matter and radiation of the universe are created via the decay of the scalar field or inflaton. Specifically, an important quantity known as the reheating temperature, $T_{r e h}$ can be found during this process. This quantity is associated to the temperature of the universe when the radiation epoch begin. A lower bound by the reheating temperature from the Big Bang Nucleosynthesis (BBN) $T_{r e h_{B B N}} \gtrsim 10^{-22} m_{p}$, where $m_{p}$ is the Planck mass, was obtained in Ref.[20]. Also, an upper bound by the reheating temperature arrives from the energy scale at the end of the inflationary period and is given by $T_{r e h} \lesssim 10^{-3} m_{p}$. We mention that the first Bayesian constraints on the single field inflationary reheating epoch from Cosmic Microwave Background data was obtained in Ref.[21], see also Ref.[22].

In this respect, afterward of the inflationary period, the inflaton field experiments coherent oscillations at the bottom of an effective potential. In this form, an essential part in the mechanism of reheating are the oscillations of the inflaton field. Nevertheless, there is in the literature some models where the effective potential does not have a minimum and then the inflaton field does not oscillate. Therefore, the mechanism of reheating does not work. In 
the literature, these models or those potentials that does not have a minimum are known as non-oscillating (NO) models [23, 24].

For this type of NO model, the first mechanism of reheating was the gravitational particle production, nevertheless this mechanism becomes inefficient, see Refs. [25, 26]. The instants preheating is another mechanism for the NO model. The instants preheating incorporates an interaction between two scalar fields; the inflaton and another field [23]. Another alternative proposal to the reheating of the universe in this type of NO model, is the introduction of curvaton field $\sigma$ [27]. It is well known also that the curvaton field explains the observed large-scale adiabatic density perturbation during the early universe [27]. In this respect, the adiabatic density perturbation is produced from the curvaton field and not from the inflaton field. In this framework, the adiabatic density perturbation is originated afterward inflation epoch and from an initial condition associates to an isocurvature perturbation[28]. Following Ref.[29], we assume the curvaton hypothesis, in which the observed value of the power spectrum the inflaton field $\mathcal{P}_{\zeta_{\phi}}$ is taken to be less than the power spectrum the curvaton $\mathcal{P}_{\zeta_{\sigma}}$. Nevertheless, we mention that in Ref.[30] was considered that the power spectrum generated by both fields are important. Another important characteristics of the curvaton is that its energy density is subdominant while the inflation takes place and becomes dominant when the inflation finish. However, the curvaton survives to the expansion of the inflationary epoch. In this respect, the curvaton reheating occurs when the curvaton decays after or before dominate its energy density. We mention that in Ref.[31] was studied a curvaton model, in which the curvaton has a nonminimal derivative coupling to gravity, see also Ref.[32].

In the framework of a non-minimal derivative coupling to gravity we would like to introduce the curvaton field as a mechanism of reheating for any effective potentials that does not minimum i.e., NO models. Therefore, the main aim of this paper is to carry out the curvaton field into the non-minimal derivative coupling to gravity for NO models and see what consequences we may derive. The outline of the paper is as follow: in section II we give a brief review of the non-minimal derivative coupling inflationary epoch. In section III we analyze the kinetic epoch for non-minimal derivative coupling. In section IV we study the dynamic of the curvaton field. Section $\mathrm{V}$ describes the curvaton decay after its domination. In section VI we analyze the decay of the curvaton field before it dominates the expansion of the universe. In section VII we study a specific example of NO model, where we consider 
an exponential potential. At the end, in section VIII includes our conclusions.

\section{NON-MINIMAL DERIVATIVE COUPLING TO GRAVITY: INFLATIONARY EPOCH}

In this section we will briefly review of the inflationary epoch in the framework the a non-minimal derivative coupling to gravity.

\section{A. Inflationary epoch: A review}

In order to describe the non-minimal derivative coupling inflationary model, we start with the corresponding field equations that must satisfy the scalar field in a flat FriedmannRobertson-Walker (FRW) background. From the action (2) we get

$$
3 H^{2}=\rho_{\phi}=\rho_{\phi}^{k i n}+\rho_{\phi}^{V}=\left(1+\frac{9 H^{2}}{M^{2}}\right) \frac{\dot{\phi}^{2}}{2}+V(\phi),
$$

and

$$
\left(1+\frac{3 H^{2}}{M^{2}}\right) \ddot{\phi}+3 H\left(1+\frac{3 H^{2}}{M^{2}}+\frac{2 \dot{H}}{M^{2}}\right) \dot{\phi}+V^{\prime}(\phi)=0,
$$

where $H:=\dot{a} / a$ is the Hubble parameter, $a=a(t)$ is the scale factor, and $V^{\prime}:=\partial V / \partial \phi$. Here the kinetic energy density is defined as $\rho_{\phi}^{k i n}=\left(1+\frac{9 H^{2}}{M^{2}}\right) \frac{\dot{\phi}^{2}}{2}$ and the energy density associated to potential energy is given by $\rho_{\phi}^{V}=V(\phi)$. The dots denote derivative with respect to the cosmological time $t$, and we shall use units such that $8 \pi G=8 \pi / m_{p}^{2}=1$.

Throughout inflation the energy density associated with the scalar field is of the order of potential energy density, and dominates over the kinetic energy, i.e., $\rho_{\phi}^{V} \gg \rho_{\phi}^{k i n}$, then the Friedmann equation can be written as[33]

$$
3 H^{2} \simeq \rho_{\phi}^{V}=V(\phi)
$$

Here we note that during inflation the condition $\rho_{\phi}^{V} \gg \rho_{\phi}^{k i n}$ or equivalently $2 V(\phi) \gg \dot{\phi}^{2}(1+$ $9 H^{2} / M^{2}$ ) coincides with first slow roll approximation analyzed in Ref.[34]. In this form, the standard condition $V(\phi) \gg \dot{\phi}^{2}$ is modified in the inflationary scenario of non-minimal derivative coupling to gravity.

On the other hand, the universe can undergo a stage of accelerated expansion only if $\ddot{a}>0$, and this condition is model-independent, otherwise the gravity decelerates the expansion. 
In this form, considering that during inflation $H^{2}>\dot{H}$ ( or equivalently $\ddot{a}>0$ ) and neglecting the acceleration of the scalar field, the equation of motion associated to the scalar field given by Eq.(6]), reduces to 33 ]

$$
3 H\left(1+\frac{3 H^{2}}{M^{2}}\right) \dot{\phi}+V^{\prime}(\phi) \simeq 0,
$$

and the velocity of the scalar field $\dot{\phi}$ becomes

$$
\dot{\phi}=-\frac{V^{\prime}}{\sqrt{3 V}}\left(1+\frac{V}{M^{2}}\right)^{-1} .
$$

Here we have used Eq.(7). We mention that the high friction limit is characterize by the condition $H^{2} \gg M^{2}$. Also, different inflationary models in this limit was developed in Ref.[35] and numerical simulations in Ref.[33]. Also we mention that this condition of high friction limit suggests the addition of new conditions for the slow-roll approximations given by $3 \dot{\phi}^{2} / 2 M^{2} \ll 1$ and $3 V(\phi) / 8 \gg 1$, as was shown in Ref.[35]. Here, the authors found that these conditions give rise to solution of the type Little Rip scenario. In the following we will not consider this high friction limit and we will study the early universe in the framework of Ref.[33].

By introducing the slow-roll parameter $\epsilon$, we get

$$
\epsilon=-\frac{\dot{H}}{H^{2}} \simeq \frac{V^{\prime 2}}{2 V^{2}\left(1+V / M^{2}\right)} .
$$

Now considering that inflation ends when the slow-roll parameter $\epsilon=1$ (or equivalently $\ddot{a}=0)$, then we can find the value of the potential $V\left(\phi=\phi_{e}\right)=V_{e}$ at the end of inflation.

On the other hand, the number of e-folds $N_{*}$ is determined by $N_{*}=\int_{t_{*}}^{t_{e}} H\left(t^{\prime}\right) d t^{\prime}$, and can be written as

$$
N_{*}=-\int_{\phi_{*}}^{\phi_{e}} \frac{V}{V^{\prime}}\left[1+\frac{V}{M^{2}}\right] d \phi .
$$

In the following, the subscripts ${ }^{\prime} *^{\prime}$ and ${ }^{\prime} e^{\prime}$ are used to indicate the time when the cosmological scale leaves the horizon during inflation and the end of the inflationary scenario, respectively.

\section{KINETIC EPOCH}

In this section we analyze the kinetic epoch of the inflaton field. It is well known that when inflation has finished the model into the 'kinetic epoch' (or 'kination', for simplicity) 
[36]. The kinetic epoch occurs at the end of inflation when almost all the energy density of inflaton field is kinetic energy. However, we mention that the kinetic epoch does not take place immediately afterward of the inflationary epoch [37].

By assuming that during this epoch the kinetic energy $\rho_{\phi}^{k i n}>\rho_{\phi}^{V} \Leftrightarrow\left(1+\frac{9 H^{2}}{M^{2}}\right) \dot{\phi}^{2} / 2>V(\phi)$ and considering that the term $V^{\prime}=\partial V / \partial \phi$, is very small compared to the non standard friction term and the acceleration of the scalar field in the field Eq.(6), then the field equations during this epoch reduce to

$$
3 H^{2}=\rho_{\phi}^{k i n} \simeq\left(1+\frac{9 H^{2}}{M^{2}}\right) \frac{\dot{\phi}^{2}}{2}
$$

and

$$
\left(1+\frac{3 H^{2}}{M^{2}}\right) \ddot{\phi}+3 H\left(1+\frac{3 H^{2}}{M^{2}}+\frac{2 \dot{H}}{M^{2}}\right) \dot{\phi} \simeq 0
$$

respectively.

From Eq.(13) we find a first integral given by

$$
\dot{\phi}=\frac{a_{k}^{3}\left(1+3 H_{k}^{2} / M^{2}\right)}{a^{3}\left(1+3 H^{2} / M^{2}\right)} \dot{\phi}_{k},
$$

and corresponds to the velocity of scalar field during the kinetic epoch. In the following, the subscription ' $\mathrm{k}$ ', labels the different quantities at the starting of this epoch.

Combining Eqs.(12) and (14), we obtain that during the kinetic epoch, the Hubble parameter in terms of the scale factor results

$$
H^{2}(a)=H_{k}^{2}\left(\frac{F(a)}{F\left(a_{k}\right)}\right)
$$

where the function $F(a)$ is given by

$$
F(a)=\frac{2^{1 / 3}\left(\frac{9 A a_{k}^{6}}{a^{6}}+1\right)}{3\left[B(a)+\sqrt{4\left(\frac{9 A a_{k}^{6}}{a^{6}}+1\right)^{3}+B(a)^{2}}\right]^{1 / 3}}+\frac{\left[B(a)+\sqrt{4\left(\frac{9 A a_{k}^{6}}{a^{6}}+1\right)^{3}+B(a)^{2}}\right]^{1 / 3}}{32^{1 / 3}}-\frac{2}{3},
$$

in which

$$
B(a)=27 \frac{A a_{k}^{6}}{a^{6}}+18\left(1-\frac{3 A a_{k}^{6}}{a^{6}}\right)-16, \quad \text { with } \quad A=\frac{\dot{\phi}_{k}^{2}\left(1+3 H_{k}^{2} / M^{2}\right)^{2}}{2 M^{2}} .
$$


From the Friedmann equation given by Eq.(12), the energy density or kinetic energy $\rho_{\phi}^{k i n}=\rho_{\phi}^{k i n}(a)$, can be written as

$$
\rho_{\phi}^{k i n}(a)=\rho_{\phi}^{k}\left(\frac{a_{k}}{a}\right)^{6}\left(\frac{1+3 H_{k}^{2} / M^{2}}{1+3 H^{2} / M^{2}}\right)^{2}\left(\frac{1+9 H^{2} / M^{2}}{1+9 H_{k}^{2} / M^{2}}\right),
$$

where the Hubble parameter $H=H(a)$ is given by Eq.(15) and $H\left(a=a_{k}\right)=H_{k}$.

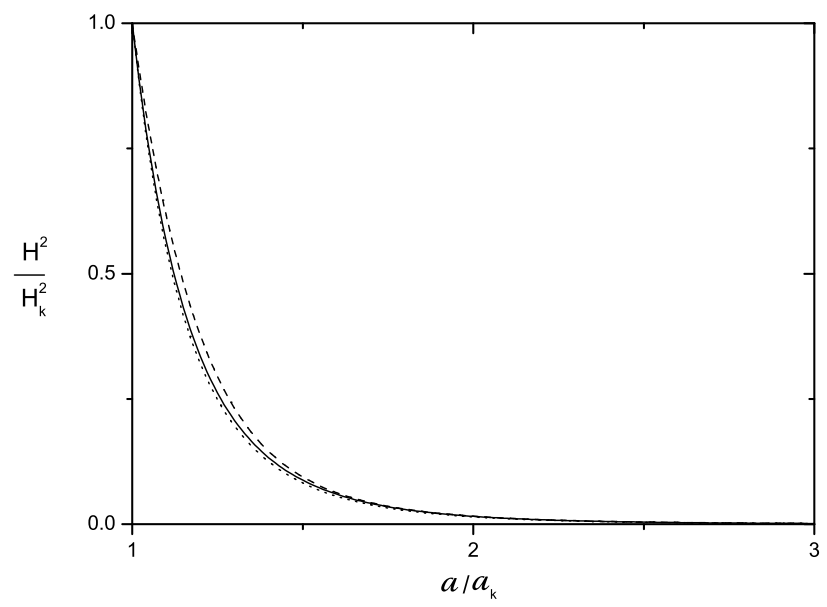

FIG. 1: Evolution of the square Hubble parameter $H^{2} / H_{k}^{2}$ versus the scale factor $a / a_{k}$, for different values of the dimensionless parameter $A$ during the kinetic epoch. The dot and dashed lines are for $A=0.1$ and $A=1$, respectively. The solid line corresponds to the standard kinetic epoch in GR, in which $H \sim a^{-3}$.

In Fig.(1) we show the evolution of dimensionless square Hubble parameter $H^{2} / H_{k}^{2}$ versus the scale factor $a / a_{k}$, for different values of the dimensionless parameter $A$ during the kinetic epoch. Here we consider the solution given by Eq.(15). In this plot we analyze two different values of the parameter $A$ and also we consider the specific case of the standard kinetic epoch in General Relativity (GR). In particular, the dot and dashed lines are for the specific values of $A=0.1$ and $A=1$, and the solid line corresponds to the standard kinetic epoch, where $H \sim a^{-3}$. From this plot we note that when we decrease the parameter $A \rightarrow 0$, the Hubble parameter during the kinetic epoch presents a small displacement with respect to the value of $H \sim a^{3}$. Also, we observe that the incorporation of the new parameter $M$ gives us a freedom that allows us to change the standard scenario of the kinetic epoch in GR. 


\section{THE DYNAMIC OF THE CURVATON}

In this section we study the dynamic of the curvaton field $\sigma$, through different epochs. From the dynamic of the field $\sigma$, we can find the constraints upon the parameter in our model in order to obtain a viable model. For the dynamic, we assume that the curvaton

field satisfies the Klein-Gordon equation with a scalar potential $U(\sigma)$ given by $U(\sigma)=\frac{m^{2} \sigma^{2}}{2}$, where the parameter $m$ corresponds to the curvaton mass.

Firstly, we assume that the inflaton field coexists with the curvaton during the inflationary scenario. However, we consider that the energy density associate to the curvaton field $\rho_{\sigma}$ is lower than the energy density of inflaton field, i.e., $\rho_{\sigma} \ll \rho_{\phi}$, such that the inflaton field $\phi$ always drives the inflationary expansion of the early universe. In the next scenario, the curvaton presents oscillations at the minimum of its effective potential $U(\sigma)$. In this respect, the dynamic of the energy density of curvaton field evolves as a non-relativistic matter and the expansion of the universe is even dominated by the inflaton field. Finally, in the last scenario the curvaton field decays into radiation, and then we recovered the Big-Bang model.

During the inflationary expansion, is considered that the curvaton mass $m \ll H_{e}$, which means domination of the inflaton field over curvaton, for more detail see Refs. [38-40]. However, in the kinetic epoch the Hubble parameter decreases until that its value becomes approximately to the curvaton mass, i.e., $H \simeq m$. From this condition and considering Eq. (15), we get

$$
\frac{m^{2}}{H_{k}^{2}} \simeq \frac{F\left(a_{m}\right)}{F\left(a_{k}\right)},
$$

here the subscript ' $m$ ' stands the quantities evaluated at time when the curvaton mass, $m \sim H$.

As it was commented above, we considered that the inflationary epoch is only driven by the inflaton field, and in order to prevent that the field curvaton produces an inflationary expansion, we consider that the energy density of the inflaton field at the times when $m \sim H$ becomes $\left.\rho_{\phi}\right|_{a_{m}}=\rho_{\phi}^{m} \gg \rho_{\sigma}$. Over inflation period, there is not substantial changes of the effective potential, and then the energy density $\rho_{\phi}^{m} \sim H^{2} \sim m^{2} \gg \rho_{\sigma} \sim U\left(\sigma_{e}\right) \sim U\left(\sigma_{*}\right)$, resulting

$$
\frac{m^{2} \sigma_{*}^{2}}{2 \rho_{\phi}^{m}}=\frac{\sigma_{*}^{2}}{6} \ll 1,
$$

or equivalently $\sigma_{*}^{2} \ll 6$. Here we note that the above condition for the value $\sigma_{*}$ coincides with the obtained in Ref.[25]. 
On the other hand, we note that at the end of inflation the energy density of the inflaton becomes subdominant over the energy of the curvaton field, i.e. $V_{e} \gg U_{e}$. In this way, considering Eq. (18) the ratio between the potential energies can be written as

$$
\frac{U_{e}}{V_{e}}=\frac{m^{2} \sigma_{*}^{2}}{6 H_{e}^{2}} \ll 1 \text { or equivalently } \frac{m}{H_{e}} \ll 1 .
$$

Here we note that the above inequality gives a lower bound for the curvaton mass $m$.

Since the Hubble parameter decreases during the expansion of the Universe, then the mass of the curvaton field becomes significant wherewith $m \simeq H$, and therefore its energy decays $\rho_{\sigma} \propto a^{-3}$ i.e., as non-relativistic matter. In this form, we write

$$
\rho_{\sigma}=\frac{m^{2} \sigma_{*}^{2}}{2} \frac{a_{m}^{3}}{a^{3}}
$$

In the following, we will consider the decay of the curvaton field in two different scenarios; when the curvaton field decays after it dominates the expansion of the Universe and when the curvaton decays before it dominates.

\section{CURVATON DECAY AFTER DOMINATION}

As we mentioned above the curvaton field decays, could take place in two different possible scenarios. In the first scenario, the curvaton dominates the cosmic expansion, i.e., the energy density of the curvaton field $\rho_{\sigma}>\rho_{\phi}$. During the expansion there must be an instant in which the energy densities of inflaton and curvaton fields are equivalents, lets say, $a=a_{e q}$. Now from the Eq.(16) and bearing in mind that $\rho_{\sigma} \propto a^{-3}$, we have

$$
\begin{aligned}
\left.\frac{\rho_{\sigma}}{\rho_{\phi}^{k i n}}\right|_{a=a_{e q}} & =\frac{m^{2} \sigma_{*}^{2}}{2} \frac{a_{m}^{3} a_{e q}^{3}}{a_{k}^{6} \rho_{\phi}^{k}}\left(\frac{1+3 H_{e q}^{2} / M^{2}}{1+3 H_{k}^{2} / M^{2}}\right)^{2}\left(\frac{1+9 H_{k}^{2} / M^{2}}{1+9 H_{e q}^{2} / M^{2}}\right) \\
& =\frac{m^{2} \sigma_{*}^{2} a_{m}^{3} a_{e q}^{3}}{6 H_{k}^{2} a_{k}^{6}}\left(\frac{1+3 H_{e q}^{2} / M^{2}}{1+3 H_{k}^{2} / M^{2}}\right)^{2}\left(\frac{1+9 H_{k}^{2} / M^{2}}{1+9 H_{e q}^{2} / M^{2}}\right)=1,
\end{aligned}
$$

where we have used the relation $3 H_{k}^{2}=\rho_{\phi}^{k}$, and also the Hubble parameter $H\left(a=a_{e q}\right)=$ $H_{e q}$, is defined as $H_{e q}=H_{k}\left[F\left(a_{e q}\right) / F\left(a_{k}\right)\right]^{1 / 2}$, see Eq.(15).

On the other hand, as the decay parameter $\Gamma_{\sigma}$ is limited from the nucleosynthesis and the Hubble parameter during this epoch is $H_{n u c l} \sim 10^{-40}$ (in units of $m_{p}$ ), then a lower bound for the parameter $\Gamma_{\sigma}$ given by $H_{n u c l} \sim 10^{-40}<\Gamma_{\sigma}$. From the other side, the condition $\rho_{\sigma}>\rho_{\phi}$ 
(curvaton decays after domination), we require $\Gamma_{\sigma}<H_{e q}$. In this way, the constraint upon the decay parameter $\Gamma_{\sigma}$, can be written as $10^{-40}<\Gamma_{\sigma}<H_{k}\left[F\left(a_{e q}\right) / F\left(a_{k}\right)\right]^{1 / 2}$.

In the following we will study the scalar perturbations related with the curvaton field $\sigma$. In order to describe the curvature perturbation from the curvaton field, we mention two possible stages. Firstly, the quantum fluctuations during the expansion of the universe are transformed into classical perturbations which have a flat spectrum. Secondly, afterward inflation the perturbations from the curvaton field are transformed into curvature perturbations and it does not need information about the nature of inflation.

While the fluctuations are inside of the horizon, these have the same differential equation that the inflaton fluctuations, wherewith the amplitude $\delta \sigma_{*}$ is given by $\delta \sigma_{*} \simeq H_{*} / 2 \pi$. Typically, the dynamics of the curvaton fluctuations outside of the horizon, are like the unperturbed curvaton field, and these fluctuations remain constant during the expansion of the universe.

In this context, the power spectrum $P_{\zeta} \sim 10^{-9}$ [4], at the time when the decay of the curvaton takes place and can be written as [41]

$$
P_{\zeta} \simeq \frac{H_{*}^{2}}{9 \pi^{2} \sigma_{*}^{2}} \simeq \frac{V_{*}}{27 \pi^{2} \sigma_{*}^{2}} \sim 10^{-9}
$$

where we have used Eq.(17).

From Eqs. (21) and (22) we write a range for the coefficient $\Gamma_{\sigma}$ given by $10^{-40}<\Gamma_{\sigma}<H_{e q}$ at the time in which curvaton field decays after domination results

$$
10^{-40}<\Gamma_{\sigma}<\frac{M}{3^{1 / 2}}\left[\frac{3 C_{1}}{2}-1+\sqrt{\left(3 C_{1} / 2-1\right)^{2}+\left(C_{1}-1\right)}\right]^{1 / 2},
$$

where the constant $C_{1} \geq \frac{8}{9}$, and is defined as

$$
C_{1}=\frac{\left(1+3 H_{k}^{2} / M^{2}\right)^{2}}{\left(1+9 H_{k}^{2} / M^{2}\right)}\left[\frac{a_{k}^{6}}{a_{m}^{3} a_{e q}^{3}}\right]\left[\frac{6 H_{k}^{2}}{m^{2} \sigma_{*}^{2}}\right] \simeq\left(1+3 H_{k}^{2} / M^{2}\right)\left[\frac{a_{k}^{6}}{a_{m}^{3} a_{e q}^{3}}\right]\left[\frac{162 \pi^{2} H_{k}^{2} P_{\zeta}}{m^{2} V_{*}}\right] .
$$

In this form, in the first scenario we find an upper limit for the reheating temperature $T_{r e h} \sim \Gamma_{\sigma}^{1 / 2}$ and then from Eq.(23)

$$
T_{r e h}<\frac{M^{1 / 2}}{3^{1 / 4}}\left[\frac{3 C_{1}}{2}-1+\sqrt{\left(3 C_{1} / 2-1\right)^{2}+\left(C_{1}-1\right)}\right]^{1 / 4} .
$$

On the other hand, assuming that the BBN temperature $T_{B B N}$ is approximately equal to $T_{B B N} \sim 10^{-22}$, and considering that the reheating temperature $T_{\text {reh }}$ occurs before the 
$\mathrm{BBN}$, then the reheating temperature satisfies, $T_{r e h}>T_{B B N}$. In this way, considering that $T_{r e h} \sim \Gamma_{\sigma}^{1 / 2}>T_{B B N}$ and Eq.(23)), we have

$$
\left(162 \pi^{2} H_{k}^{2} P_{\zeta}\right)\left(1+\frac{3 H_{k}^{2}}{M^{2}}\right)\left(\frac{a_{k}^{6}}{a_{m}^{3} a_{e q}^{3}}\right) \frac{\left(1+9 T_{B B N}^{4} / M^{2}\right)}{\left(1+3 T_{B B N}^{4} / M^{2}\right)^{2}}>m^{2} V_{*} .
$$

However, we note that the curvaton decays occurs before the electroweak scale, since the baryogenesis is situated below the electroweak scale, then the quantity $V_{*}^{1 / 4} \sim \sqrt{m_{e w} m_{p}} \sim$ $10^{10.5} \mathrm{GeV}$, in which the electroweak scale $m_{\text {ew }} \sim 1 \mathrm{TeV}[42,43]$. In this way, the square of the Hubble parameter satisfied

$$
H_{*}^{2} \simeq \frac{V_{*}}{3} \sim 10^{-32}
$$

recalled that $8 \pi / m_{p}^{2}=1$. Now we note that if the curvaton decays before the electroweak scale, then from Eqs.(25) and (26) we obtain an upper limit for the mass of the curvaton field given by

$$
10^{26}\left(1+\frac{3 H_{k}^{2}}{M^{2}}\right)\left(\frac{a_{k}^{6}}{a_{m}^{3} a_{e q}^{3}}\right) \frac{H_{k}^{2}}{\left(1+3 T_{B B N}^{4} / M^{2}\right)}>m^{2} .
$$

Here, we have used that $P_{\zeta} \sim 10^{-9}$.

\section{CURVATON DECAY BEFORE DOMINATION}

In this section we regard that the curvaton $\sigma$ decays before it dominates the expansion of the universe. In this context, the mass of the curvaton $m$, is non-negligible when is contrasted with the Hubble parameter $H$, and then we can consider that the curvaton mass $m \sim H$. On the other hand, if the curvaton field decays at a time when $\Gamma_{\sigma}=H\left(a_{d}\right)=H_{d}$, where ' $d$ ' denotes the quantities at the time when the curvaton decays, then from Eq.(15) we have

$$
\Gamma_{\sigma}=H_{d}=H_{k} \sqrt{\frac{F\left(a_{d}\right)}{F\left(a_{k}\right)}} .
$$

In this scenario, the curvaton field $\sigma$ should decay after that the mass of the curvaton $m \sim H$, satisfying the condition $\Gamma_{\sigma}<m$. However, also the curvaton field $\sigma$ should decay before that it dominate the expansion of the universe, in which $\Gamma_{\sigma}>H_{e q}$. In this form, considering Eq.(21) we get

$$
\frac{M}{3^{1 / 2}}\left[\frac{3 C_{1}}{2}-1+\sqrt{\left(3 C_{1} / 2-1\right)^{2}+\left(C_{1}-1\right)}\right]^{1 / 2}<\Gamma_{\sigma}<m .
$$

Recalled that the curvaton field decays at the time when $\rho_{\sigma}<\rho_{\phi}$. 
Following Refs. [41, 44] the Bardeen parameter $P_{\zeta}$, is given by

$$
P_{\zeta} \simeq \frac{r_{d}^{2}}{16 \pi^{2}} \frac{H_{*}^{2}}{\sigma_{*}^{2}}, \quad \text { where } \quad r_{d}=\left.\frac{\rho_{\sigma}}{\rho_{\phi}}\right|_{a=a_{d}},
$$

in which the parameter $r_{d}$ corresponds to the ratio between the curvaton and the inflaton energy densities, measured at the time in which the curvaton decay takes place.

Considering that the energy density of the curvaton decays as non-relativistic matter i.e., $\rho_{\sigma} \propto a^{-3}$, and rewritten the energy density $\rho_{\phi}$ as

$$
\rho_{\phi}(a)=\rho_{\phi}^{k}\left(\frac{a_{k}}{a}\right)^{6} \frac{K(a)}{K\left(a_{k}\right)}
$$

where the new functions $K(a)$ is defined as

$$
K(a)=\left(\frac{1+3 H_{k}^{2} / M^{2}}{1+3 \frac{F(a) H_{k}^{2}}{F\left(a_{k}\right) M^{2}}}\right)^{2}\left(\frac{1+9 \frac{F(a) H_{k}^{2}}{F\left(a_{k}\right) M^{2}}}{1+9 H_{k}^{2} / M^{2}}\right)
$$

then the ratio $r_{d}$, results

$$
r_{d}=\left.\frac{\rho_{\sigma}}{\rho_{\phi}}\right|_{a=a_{d}}=\frac{m^{2} \sigma_{*}^{2}}{6} \frac{a_{m}^{3} a_{d}^{3}}{H_{k}^{2} a_{k}^{6}} \frac{K\left(a_{k}\right)}{K\left(a_{d}\right)},
$$

or equivalently using Eq.(28) the ratio $r_{d}$ can be rewritten as

$$
r_{d}=\frac{m^{2} \sigma_{*}^{2}}{6} \frac{a_{m}^{3} a_{d}^{3}}{H_{k}^{2} a_{k}^{6}}\left(\frac{1+3 \Gamma_{\sigma}^{2} / M^{2}}{1+3 H_{k}^{2} / M^{2}}\right)^{2}\left(\frac{1+9 H_{k}^{2} / M^{2}}{1+9 \Gamma_{\sigma}^{2} / M^{2}}\right) .
$$

From Eqs.(30) and (32), we find that the parameter $\Gamma_{\sigma}$ can be written as

$$
\Gamma_{\sigma} \approx \frac{M}{\sqrt{3}}\left[\frac{24 \pi H_{k}^{2}}{m^{2} H_{*} \sigma_{*}} \sqrt{P_{\xi}}\left(\frac{a_{k}^{6}}{a_{m}^{3} a_{d}^{3}}\right)\left(1+3 H_{k}^{2} / M^{2}\right)-1\right]^{1 / 2} .
$$

In this way, in the second scenario we find that the reheating temperature using Eq.(33) results

$$
T_{r e h} \sim \frac{M^{1 / 2}}{3^{1 / 4}}\left[\frac{24 \pi H_{k}^{2}}{m^{2} H_{*} \sigma_{*}} \sqrt{P_{\xi}}\left(\frac{a_{k}^{6}}{a_{m}^{3} a_{d}^{3}}\right)\left(1+3 H_{k}^{2} / M^{2}\right)-1\right]^{1 / 4} .
$$

Also, considering Eq.(29), we obtain that the condition for the scalar field $\sigma_{*}$ becomes

$$
\sigma_{*}<\frac{24 \pi H_{k}^{2}}{m^{2} H_{*} \sigma_{*}} \sqrt{P_{\xi}}\left(\frac{a_{k}^{6}}{a_{m}^{3} a_{d}^{3}}\right)\left(1+3 H_{k}^{2} / M^{2}\right)\left[\frac{3 C_{1}}{2}+\sqrt{\left(3 C_{1} / 2-1\right)^{2}+\left(C_{1}-1\right)}\right]^{-1} \ll 6 .
$$

Here we have considered that $\sigma_{*} \ll 6$, from the dynamic of the curvaton (see section IV). 


\section{AN EXAMPLE: EXPONENTIAL POTENTIAL}

In the following we study an exponential potential as an example of NO model. The exponential potential is defined as

$$
V(\phi)=V_{0} e^{-\alpha \phi}
$$

where $V_{0}$ and $\alpha$ are two positive parameters. This kind of potential was found in power law inflation in which the scale factor $a(t) \propto t^{p}$, where the exponent $p>1$ [45]. Also the exponential potential has been studied in the string theory and tachyonic cosmologies [46]. Another NO potentials can be found in Ref.[47].

From the exponential potential and considering Eq.(9), we obtain that the scalar potential as function of the time (or $\phi(t))$, becomes

$$
V(t)=V_{0} e^{-\alpha \phi(t)}=\left[\sqrt{\frac{M^{4}}{4}\left(C+\frac{\alpha^{2}}{2 \sqrt{3}} t\right)^{2}+M^{2}}-\frac{M^{2}}{2}\left(C+\frac{\alpha^{2}}{2 \sqrt{3}} t\right)\right]^{2},
$$

where the integration constant is defined as $C=\frac{e^{\alpha \phi_{0} / 2}}{\sqrt{V_{0}}}-\frac{1}{M^{2}} \sqrt{V_{0}} e^{-\alpha \phi_{0} / 2}$, in which $\phi(t=$ $0)=\phi_{0}$.

From the slow-roll parameter $\epsilon$, we get

$$
\epsilon=-\frac{\dot{H}}{H^{2}}=\frac{V^{\prime 2}}{2 V^{2}\left(1+V / M^{2}\right)}=\frac{\alpha^{2}}{2\left(1+V / M^{2}\right)} .
$$

Now considering that inflation ends when the slow-roll parameter $\epsilon=1$ (or equivalently $\ddot{a}=0$ ), then we find that the value of the potential $V_{e}$ at the end of inflation results

$$
V_{e}=M^{2}\left(\frac{\alpha^{2}}{2}-1\right)
$$

which implies that the parameter $\alpha>\sqrt{2}$, since the value of the potential $V_{e}>0$. Also, we obtain that the number of e-folds $N_{*}$ results

$$
N_{*}=\frac{1}{\alpha}\left[\phi_{e}-\phi_{*}\right]+\frac{1}{\alpha^{2} M^{2}}\left(V_{*}-V_{e}\right)=\frac{1}{\alpha^{2}}\left[\ln \left(V_{*} / V_{e}\right)+\frac{1}{M^{2}}\left(V_{*}-V_{e}\right)\right] .
$$

In Fig.(2) we show the parameter $M$ versus the number of e-folds $N_{*}$, for different values of the parameter $\alpha$ associated to the exponential potential. Here we studied three different values of the parameter $\alpha$. In order to write down values that relate the parameter $M$ and 


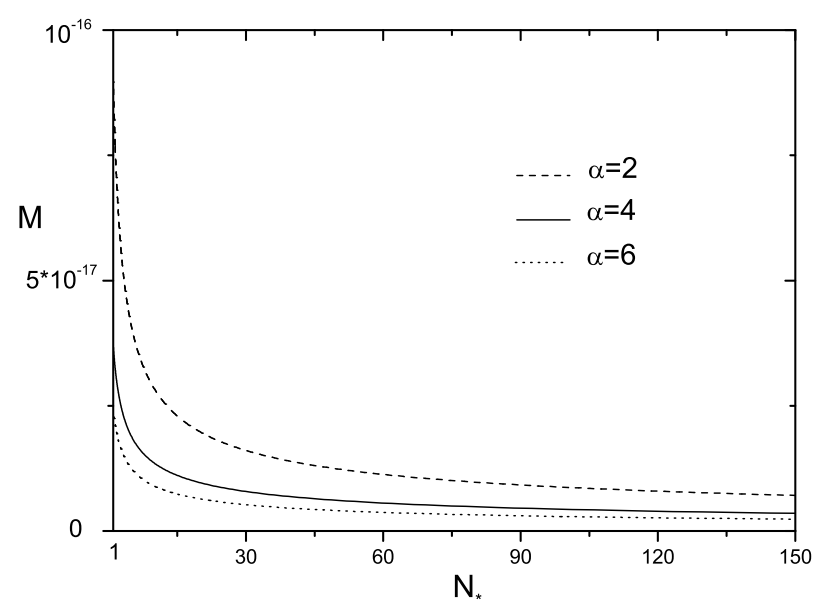

FIG. 2: The parameter $M$ as a function of the number of e-folds $N_{*}$, for different values of the parameter $\alpha$. The dot, solid and dashed lines are for the values $\alpha=6, \alpha=4$ and $\alpha=2$. Here we have used that $V_{*}=3 \times 10^{-32}$.

the number of e-folds, we considering the relation given by Eq.(40). Also, we have taken the value $V_{*}=3 * 10^{-32}$ from Eq.(26). In particular, the dot, solid and dashed lines are for the specific values of $\alpha=6$ and $\alpha=4$, and $\alpha=2$, respectively. We note that when we increase the value of the parameter $\alpha$ (recall that $\alpha>\sqrt{2}$ ) the number of e-folds $N_{*}$ decreased and also the value of the parameter $M$. Also from the plot we observe that the value of the parameter $M<10^{-16}$ is well supported by the the number of e-folds $N_{*} \gtrsim 60$.

From dynamics of the curvaton, we find that at the end of inflation the energy density of the inflaton becomes subdominant over the energy of the curvaton field, i.e. $V_{e} \gg U_{e}$. In this way, considering Eqs.(18) and (39), the ratio between the potential energies can be written as

$$
\frac{U_{e}}{V_{e}}=\frac{m^{2} \sigma_{*}^{2}}{6 H_{e}^{2}} \ll \frac{m^{2}}{H_{e}^{2}}=\frac{3 m^{2}}{M^{2}\left(\alpha^{2} / 2-1\right)} \ll 1,
$$

and then the ratio $m / M$, satisfied

$$
\frac{m}{M} \ll \sqrt{\frac{\left(\alpha^{2} / 2-1\right)}{3}} .
$$

Here, we note that from the dynamic of the curvaton, we obtain an upper bound for the rate $m / M$. 


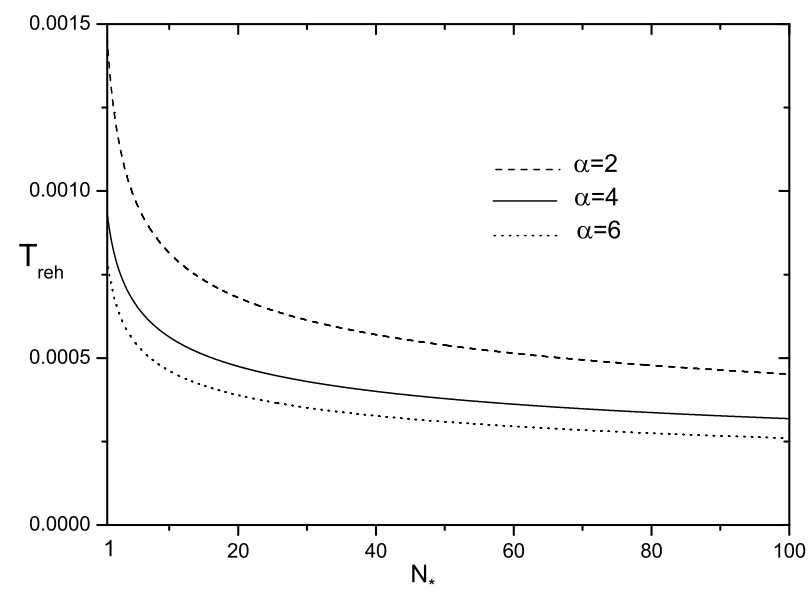

FIG. 3: The reheating temperature $T_{r e h}$ as a function of the number of e-folds $N_{*}$, for different values of the parameter $\alpha$ when the curvaton decays after it dominates the expansion of the universe. The dot, solid and dashed lines are for the values $\alpha=6, \alpha=4$ and $\alpha=2$. Here we have used the values $M=10^{-17}, m=10^{-20}, H_{k}=10^{-17}$ and $a_{k}^{2} /\left(a_{m} a_{e q}\right)=10^{-3}$.

In Fig.(3) we show the reheating temperature $T_{r e h}$ (in units of $m_{p}$ ) on the number of e-folds $N_{*}$, when the curvaton field decays after it dominates the expansion of the universe. Here we have used three different values of the parameter $\alpha$ associated to the exponential potential, where the dot, solid and dashed lines are for the values $\alpha=6, \alpha=4$ and $\alpha=2$. From Eq.(24) we can obtain the reheating temperature $T_{r e h}$ as a function of the potential $V_{*}$, i.e., $T_{r e h}=T_{r e h}\left(V_{*}\right)$ and together with Eqs.(39) and (40), we numerically find the parametric plot of the curve $T_{r e h}=T_{r e h}(N)$. This method to determine the reheating temperature in terms of the number of e-folds $N_{*}$ during the evolution of the universe, was introduced in Ref.[48].

In this plot we have considered the values $M=10^{-17}, m=10^{-20}$ from relation given by Eq.(42), $H_{*} \simeq 10^{-16}>H_{k}=10^{-17}$ see Eq.(26), and considering that $a_{k}<a_{m}<a_{e q}$ then we have taken $a_{k}^{2} /\left(a_{m} a_{e q}\right)=10^{-3}$. We observe that the curves $T_{r e h}=T_{r e h}(N)$ give an upper limit for the reheating temperature, when the curvaton decays after domination in the case of an exponential potential. Also we note that when we increase the value of $\alpha$, the reheating temperature $T_{\text {reh }}$ decreases to values $T_{r e h}<10^{-3}$ for $N_{*} \simeq 60$. Here we note that this upper limit for the reheating temperature is similar to the GUT scale, where the 


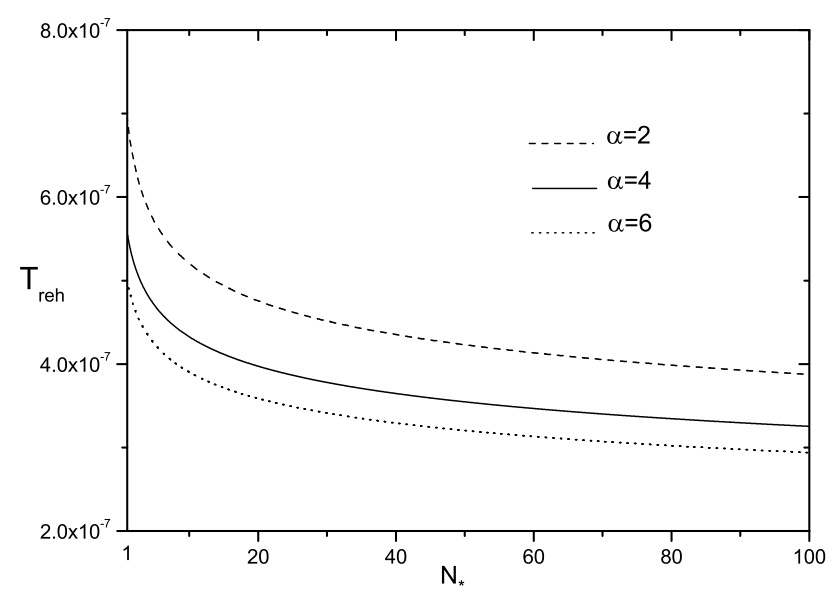

FIG. 4: The reheating temperature $T_{r e h}$ as a function of the number of e-folds $N_{*}$, for different values of the parameter $\alpha$ when the curvaton decays before it dominates the expansion of the universe. The dot, solid and dashed lines are for the values $\alpha=6, \alpha=4$ and $\alpha=2$. As before, we have used the values $M=10^{-17}, m=10^{-20}, H_{k}=10^{-17}, \sigma_{*}=10^{-2}$, and $\left[a_{k}^{2} /\left(a_{m} a_{d}\right)\right]^{3}=10^{-10}$.

temperatute $T_{r e h_{G U T}} \lesssim 10^{-3}$ (in units of $m_{p}$ ). Also, we observe that this upper limit in the $T_{r e h}$, is similar to that found in Ref.[40].

In Fig.(41) we show the reheating temperature $T_{r e h}$ versus the number of e-folds $N_{*}$ when the curvaton field decays before it dominates the expansion of the universe. As before, we have used three different values of the parameter $\alpha$, where the dot, solid and dashed lines are for the values $\alpha=6, \alpha=4$ and $\alpha=2$. From Eq.(34) we can find the reheating temperature $T_{r e h}$ as a function of the potential $V_{*}$ and together with Eqs.(39) and (40), we numerically obtain the parametric plot $T_{r e h}=T_{r e h}(N)$. As before, in this plot we have used the values $M=10^{-17}, m=10^{-20}$ and $H_{*} \simeq 10^{-16}>H_{k}=10^{-17}$. Also, we have considered that $a_{k}^{6} /\left(a_{m} a_{d}\right)^{3}=10^{-10}$ and $\sigma_{*}=10^{-2}$. We note from Fig.(44) that when we increase the value of the parameter $\alpha$, the reheating temperature $T_{r e h}$ decreases to values $T_{\text {reh }}<10^{-6}$ for $N_{*} \gtrsim 60$. In particular for the case in which $N_{*}=60$ and $\alpha=2$, we obtain that the reheating temperature $T_{\text {reh }} \approx 4 \times 10^{-7}$, for the value $\alpha=4$ corresponds to $T_{\text {reh }} \approx 3.5 \times 10^{-7}$, and for the value $\alpha=6$ corresponds to $T_{\text {reh }} \approx 3 \times 10^{-7}$. It follows that one must increase the reheating temperature by three orders of magnitude to have a $T_{r e h}$ close to the $T_{r e h_{G U T}}$. 


\section{CONCLUSIONS}

We have analyzed in general form and in detail the curvaton mechanism of reheating into the NO models in the context of the non-minimal derivative coupling to gravity. In this framework, we have considered that the curvaton field drives the reheating the Universe as well as for the curvature perturbations. Also, we have studied the kinetic epoch in our model and we obtained the evolution of the Hubble parameter and kinetic energy expressed by Eqs. (15) and (16), respectively. In explaining the curvaton reheating we have studied two possible scenarios: i) The curvaton field decays after it dominates the cosmic expansion of the universe and ii) the curvaton decays before it dominates the expansion. During the first scenario, we have found an upper limit for the parameter $\Gamma_{\sigma}$ or equivalently an upper limit for the reheating temperature specified by Eq.(24). For the second scenario, we have obtained an approximate value for the temperature expressed by Eq.(34).

As a specific example of NO model, we have studied an exponential potential. For this potential we have considered the method of constraining the reheating temperature indirectly from the inflationary period through the number of e-folds i.e., $T_{r e h}=T_{r e h}\left(N_{*}\right)$. During the first scenario when the curvaton decays after it dominates the expansion, we have found that for values of $\alpha>\sqrt{2}$, the reheating temperature is approximately $T_{r e h}<10^{-3}$ (in units of $m_{p}$ ) as an upper bound. In the second scenario when the curvaton decays before it dominates, we have obtained that the $T_{r e h}<10^{-6}$ for values of $\alpha>\sqrt{2}$. We noted that these values for the temperatures are similar to those found in Ref. [40].

\section{Acknowledgments}

R. H. and J. S. were supported by the COMISION NACIONAL DE CIENCIAS Y TECNOLOGIA through FONDECYT Grant $\mathrm{N}_{0}$ 1130628. R. H. was partially supported by DI-PUCV Grant $\mathrm{N}_{0} 123724$.

[1] A. Guth , Phys. Rev. D 23, 347 (1981); A.A. Starobinsky, Phys. Lett. B 91, 99 (1980); A.D. Linde, Phys. Lett. B 108, 389 (1982); idem Phys. Lett. B 129, 177 (1983); A. Albrecht and 
P. J. Steinhardt, Phys. Rev. Lett. 48,1220 (1982); K. Sato, Mon. Not. Roy. Astron. Soc. 195, 467 (1981).

[2] V.F. Mukhanov and G.V. Chibisov, JETP Letters 33, 532(1981); S. W. Hawking, Phys. Lett. B 115, 295 (1982); A. Guth and S.-Y. Pi, Phys. Rev. Lett. 49, 1110 (1982); A. A. Starobinsky, Phys. Lett. B 117, 175 (1982); J.M. Bardeen, P.J. Steinhardt and M.S. Turner, Phys. Rev.D 28, 679 (1983).

[3] G. Smoot, et al. Astrophys. J. Lett. 396, L1 (1992).

[4] P. A. R. Ade et al. [Planck Collaboration], arXiv:1502.02114 [astro-ph.CO].

[5] P. Jordan, Z. Phys. 157, 112 (1959); C. Brans and R.H. Dicke, Phys. Rev. 124, 925 (1961).

[6] L. Randall and R. Sundrum. Phys. Rev. Lett., 83, 3370, (1999); L. Randall and R. Sundrum, Phys.Rev.Lett. 83, 4690 (1999).

[7] G. Pulgar, J. Saavedra, G. Leon and Y. Leyva, JCAP 1505, no. 05, 046 (2015).

[8] L. Amendola, Phys. Lett. B 301, 175 (1993).

[9] S. Capozziello, G. Lambiase and H. J. Schmidt, Annalen Phys. 9, 39 (2000).

[10] G. W. Horndeski, Int. J. Theor. Phys. 10, 363 (1974).

[11] S. V. Sushkov, Phys. Rev. D 80, 103505 (2009).

[12] E. N. Saridakis and S. V. Sushkov, Phys. Rev. D 81, 083510 (2010).

[13] A. A. Starobinsky, S. V. Sushkov and M. S. Volkov, JCAP 1606 (2016) no.06, 007.

[14] C. Germani and A. Kehagias, Phys. Rev. Lett. 105 , 011302 (2010); C. Germani and A. Kehagias, Phys. Rev. Lett. 106 , 161302 (2011).

[15] J. B. Dent, S. Dutta, E. N. Saridakis and J. Q. Xia, JCAP 1311, 058 (2013).

[16] S. F. Daniel and R. R. Caldwell, Class. Quant. Grav. 24, 5573 (2007); E. N. Saridakis and S. V. Sushkov, Phys.Rev. D 81, 083510 (2010); S. Sushkov, Phys. Rev. D 85, 123520 (2012).

[17] M. A. Skugoreva, S. V. Sushkov, and A. V. Toporensky, Phys. Rev. D 88, 083539 (2013); A. De Felice and S. Tsujikawa, Phys. Rev. D 84, 083504 (2011); R. Jinno, K. Mukaida and K. Nakayama, JCAP 1401, 031 (2014); Y. S. Myung, T. Moon and B. H. Lee, JCAP 1510, no. 10, 007 (2015); Y. S. Myung and T. Moon, arXiv:1601.03148 [gr-qc].

[18] C. Germani and A. Kehagias, JCAP 1005, 019 (2010) [JCAP 1006, E01 (2010)]; F. Darabi and A. Parsiya, Class. Quant. Grav. 32, no. 15, 155005 (2015).

[19] L. Kofman and A. Linde, JHEP 0207, 004 (2002).

[20] S. Hannestad, Phys. Rev. D 70, 043506 (2004). 
[21] J. Martin and C. Ringeval, Phys. Rev. D 82, 023511 (2010).

[22] M. J. Mortonson, H. V. Peiris and R. Easther, Phys. Rev. D 83, 043505 (2011); R. Easther and H. V. Peiris, Phys. Rev. D 85, 103533 (2012); M. A. Amin, R. Easther, H. Finkel, R. Flauger and M. P. Hertzberg, Phys. Rev. Lett. 108, 241302 (2012); J. Martin, C. Ringeval and V. Vennin, Phys. Rev. Lett. 114, no. 8, 081303 (2015).

[23] G. Felder, L. Kofman and A. Linde, Phys. Rev. D 60, 103505 (1999).

[24] B. Feng and M. Li, Phys. Lett. B 564, 169 (2003).

[25] A. R. Liddle and L. A. Ureña-López, Phys. Rev. D 68, 043517 (2003).

[26] M. Sami, P. Chingangbam and T. Qureshi, Phys. Rev. D 66, 043530 (2002).

[27] D. H. Lyth and D. Wands, Phys. Lett. B 524, 5 (2002).

[28] S. Mollerach, Phys. Rev. D 42, 313 (1990).

[29] K. Dimopoulos and D. H. Lyth, Phys. Rev. D 69, 123509 (2004); M. Beltran, Phys. Rev. D 78, 023530 (2008).

[30] K. Dimopoulos, D. H. Lyth, A. Notari and A. Riotto, JHEP 0307, 053 (2003); D. Langlois and F. Vernizzi, Phys. Rev. D 70, 063522 (2004).

[31] K. Feng, T. Qiu and Y. S. Piao, Phys. Lett. B 729, 99 (2014).

[32] K. Feng and T. Qiu, Phys. Rev. D 90, no. 12, 123508 (2014).

[33] S. Tsujikawa, Phys. Rev. D 85, 083518 (2012).

[34] J. Matsumoto and S. V. Sushkov, JCAP 1511, no. 11, 047 (2015).

[35] N. Yang, Q. Gao and Y. Gong, arXiv:1504.05839 [gr-qc]; B. Gumjudpai and P. Rangdee, Gen. Rel. Grav. 47, no. 11, 140 (2015).

[36] M. Joyce and T. Prokopec, Phys. Rev. D 57, 6022 (1998).

[37] Z. K. Guo, Y. S. Piao, R. G. Cai and Y. Z. Zhang, Phys. Rev. D 68, 043508 (2003).

[38] J. C. Bueno Sanchez and K. Dimopoulos, JCAP 0711, 007 (2007); S. del Campo and R. Herrera, Phys. Rev. D 76, 103503 (2007); S. del Campo, R. Herrera, J. Saavedra, C. Campuzano and E. Rojas, Phys. Rev. D 80, 123531 (2009); E. I. Guendelman and R. Herrera, Gen. Rel. Grav. 48, no. 1, 3 (2016).

[39] M. Postma, Phys. Rev. D 67, 063518 (2003).

[40] C. Campuzano, S. del Campo and R. Herrera, Phys. Lett. B 633, 149 (2006); idem, Phys. Rev. D 72, 083515 (2005); idem, JCAP 0606, 017 (2006).

[41] S. Mollerach, Phys. Rev. D 42, 313 (1990). 
[42] K. Dimopoulos, Phys. Lett. B 634, 331 (2006).

[43] K. Dimopoulos, G. Lazarides, D. Lyth and R. Ruiz de Austri, Phys. Rev. D 68, 123515 (2003).

[44] D. H. Lyth, C. Ungarelli and D. Wands, Phys. Rev. D 67, 023503 (2003).

[45] F. Lucchin and S. Matarrese, Phys. Rev. D 32, 1316 (1985).

[46] M. Sami, P. Chingangbam and T. Qureshi, Phys. Rev. D 66, 043530 (2002); A. Sen, Mod. Phys. Lett. A 17, 1797 (2002).

[47] J. D. Barrow, Class. Quant. Grav. 13, 2965 (1996); J. D. Barrow and N. J. Nunes, Phys. Rev. D 76043501 (2007); S. del Campo and R. Herrera, Phys. Lett. B 660, 282 (2008); S. del Campo, E. I. Guendelman, A. B. Kaganovich, R. Herrera and P. Labrana, Phys. Lett. B 699, 211 (2011); R. Herrera, M. Olivares and N. Videla, Eur. Phys. J. C 73, no. 1, 2295 (2013); S. Campo, C. Gonzalez and R. Herrera, Astrophys. Space Sci. 358, no. 2, 31 (2015); R. Herrera, N. Videla and M. Olivares, Eur. Phys. J. C 76, no. 1, 35 (2016).

[48] J. Mielczarek, Phys. Rev. D 83, 023502 (2011). 